\title{
Hemostasis Efficacy and Mechanism of Cold Atmospheric Air Plasma
}

\author{
Spencer Kuo \\ Department of Electrical \& Computer Engineering, New York University-Tandon School of Engineering, Brooklyn, USA \\ Email: spk259@nyu.edu
}

How to cite this paper: Kuo, S. (2021) Hemostasis Efficacy and Mechanism of Cold Atmospheric Air Plasma. Open Journal of Emergency Medicine, 9, 66-83. https://doi.org/10.4236/ojem.2021.93009

Received: June 16, 2021

Accepted: August 1, 2021

Published: August 4, 2021

Copyright (c) 2021 by author(s) and Scientific Research Publishing Inc. This work is licensed under the Creative Commons Attribution International License (CC BY 4.0).

http://creativecommons.org/licenses/by/4.0/ (c) (i) Open Access

\begin{abstract}
The capacity of a cold atmospheric-pressure air plasma (CAAP) device for advanced first aid is presented. Using swine as an animal model, two trials: 1) a large, curved cut in hindquarters area and 2) amputation of a front leg, were performed. Cold atmospheric-pressure air plasma effluent, which carries reactive oxygen species (ROS) atomic oxygen (OI), is applied for wound treatments. Swift hemostasis of the wounds by the CAAP treatment was demonstrated. The pressure applied by a finger on the cut arteries in trial 1 and the tourniquet applied in trial 2 could be removed immediately after the treatment and there was no re-bleed in both cases. CAAP hemostasis mechanism was explored via in-vitro tests. The tests on sodium citrate mixed blood-droplet samples show that 1) the heat delivered by the CAAP has no impact on the observed clot formation, 2) plasma effluent activates platelets to promote coagulation state and cascade, and 3) the degree of clotting increases with the total amount of applied OI by means of the CAAP effluent. It took only $16 \mathrm{~s}$ of the CAAP treatment to reach full clotting, which was considerably shortened from the natural clotting time of about 25 minutes. The tests on smeared blood samples show that the reduction of the platelet count and the increase of RBC count are proportional to the amount of applied OI. A plausible CAAP hemostasis mechanism is concluded from the in vitro test results and the animal model trials.
\end{abstract}

\section{Keywords}

Cold Atmospheric Air Plasma, Cold Atmospheric Plasma, Reactive Species, Reactive Oxygen Species, Atomic Oxygen, Trauma, Hemorrhage, Hemostasis, Animal Model, Swine, Blood Coagulation, Clotting

\section{Introduction}

The outcome of a hemorrhage varies, depending on its severity; it can range 
from no apparent damage to severe disability [1] [2] [3] [4]. Severe bleeding, in critical situations, can be life threatening if not treated swiftly [1] [4] [5]; for example, it accounts for $30 \%$ to $40 \%$ of all fatalities on battlefields [2], second only to central nervous system injury. Ways of controlling major bleeding can prevent hemorrhagic death and timely stopping hemorrhage can also relieve symptoms and prevent complications [1]-[13].

Blood coagulation involves platelet activation and coagulation cascade. After wound is bleeding, the vasoconstriction is taken place and oxidants are released in the vascular lumen to enhance platelet agglomeration at the wound location to act on blood clotting. Moreover, oxidants fragment platelets to form a fibrin clot which plugs broken blood vessels for hemostasis.

Ambrosio et al. [14] suggested that reactive oxygen metabolites might affect thrombus formation within the vasculature. In addition, hydrogen peroxide $\left(\mathrm{H}_{2} \mathrm{O}_{2}\right)$, a non-radical yet oxidant species, is known to play a special role in platelet agglomeration [15] [16]. Studies have shown that platelets are a prime target for oxidants produced or released in the vascular lumen. Oxidants can affect several key steps of platelet function to indirectly enhance platelet agglomeration through local increases in platelet-activating factor (PAF) [17]. Furthermore, oxidants promote de novo synthesis of tissue factor pro-coagulant activity [18].

Plasma can effectively convert electromagnetic energy into kinetic energy of electrons, which is needed for exciting and dissociating molecular oxygen to produce chemically reactive oxygen species (ROS), including molecular oxygen in metastable states and atomic oxygen. Atmospheric pressure plasmas, used in medical applications, can be generated by various electric discharges [19] [20] [21] [22]; however, plasmas must be generated in a non-equilibrium state, so that the electron kinetic energy, gained from the electric source, is better used for producing reactive species, rather than for heating the plasma effluent; keeping plasma in low temperature. The reactive species carried by the plasma effluent can trigger biochemical reactions, for example, atomic oxygen interacts with $\mathrm{H}_{2} \mathrm{O}$, similar oxidants to those released in the vascular lumen are generated to speed up blood clotting and clot formation, and reactive species furnish biocidal effects for sterilization applications, and promote wound healing [23]-[33].

The flux of the reactive species carried by the plasma is somehow proportional to the discharge current, the concentration of $\mathrm{O}_{2} / \mathrm{N}_{2}$ in the working gas, and the percentage of the energetic electrons in the electron plasma. Noble gases and noble gasses mixed with $\mathrm{N}_{2} / \mathrm{O}_{2}$ have been used to lower the plasma temperature [20] [22] [23] [24], but the tradeoff is lowering the intensities of generated reactive species.

On the other hand, using ambient air as the working gas, chemically reactive oxygen species (ROS) and nitrogen species (RNS), including molecular oxygen/nitrogen in metastable states and atomic oxygen/nitrogen, are generated directly in the plasma. Air flow and/or magnetic field have been introduced in devices to generate cold atmospheric air plasma (CAAP) spray [34], where the 
discharge paths are elongated by the airflow, and rotating in the magnetic field to keep electric discharge diffusive. In other words, the arc column dragged by the gas flow evolves to gliding arc mode. The non-equilibrium stage starts when the length of the gliding arc exceeds a critical value. The magnetic field rotates the gliding arc, making it diffusive and prolonging the non-equilibrium state. A periodic voltage source also works to maintain the non-equilibrium state. The advantage of running the air discharge in low voltage high current diffusive gliding arc mode is that the power supply can be compact and additional gas tank is not necessary, which make it easy to design a practical portable CAAP device [34].

The present work demonstrates the capacity of a CAAP spray on bleeding control in critical situations, via in-vivo trial, using swine as an animal model, and explores its blood coagulation mechanism via in-vitro tests on blood-droplet samples and on smeared blood samples. Blood cell staining, optical microscopy, and histological examination for morphological studies of untreated and post-exposed blood samples were performed. The electric characteristics of the discharge for plasma generation and the spatial distribution of the $777.4 \mathrm{~nm}$ emissions of the plasma spray, a signature of the generation and distribution of atomic oxygen in the plasma effluent, are presented in Section 2. In-vivo trials, using swine as animal model, to demonstrate the capacity of CAAP in bleeding control are presented in Section 3. In-vitro tests and the results of analyses, as the basis of plasma coagulation mechanism, are presented in Section 4. The correlation of these results evidences a plausible CAAP hemostasis mechanism which is summarized in Section 5. Discussions and conclusions are given in Section 6.

\section{Plasma Spray Generator}

A non-equilibrium air plasma, generated in open air with large spatial extent by a plasma spray generator as shown in Figure 1(a), is applied to wounds for swift hemostasis.
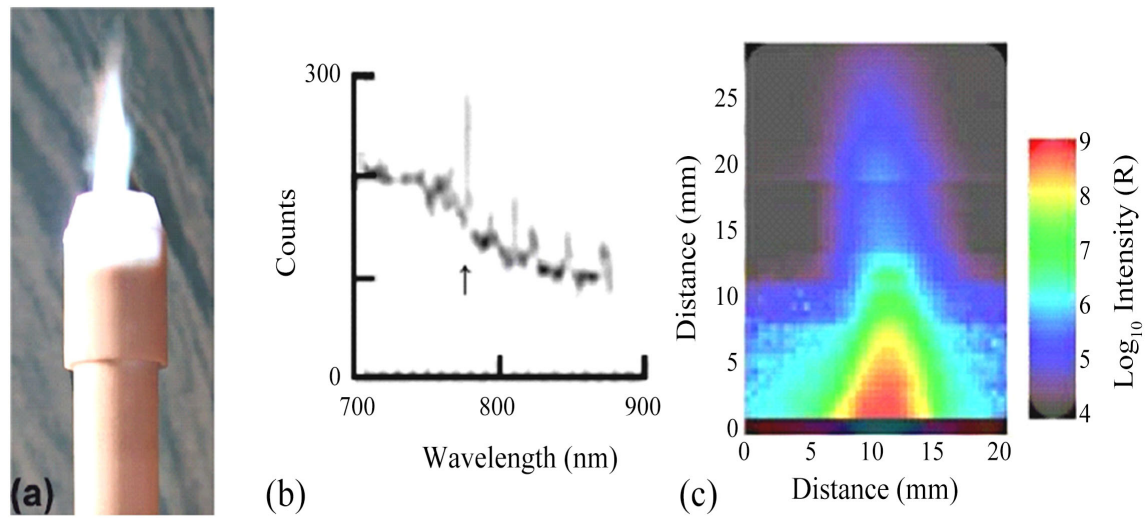

Figure 1. (a) Cold atmospheric air plasma generated by a plasma spray generator; (b) photon counts of the emission lines from 700 to $900 \mathrm{~nm}$, the arrow is located at $777.4 \mathrm{~nm}$ attributed to the $5 \mathrm{P}$ state of atomic oxygen; (c) 2-D spatial distribution of $777.4 \mathrm{~nm}$ radiation intensity of the plasma spray. 
At atmospheric pressure, the air discharge between the circular electrodes is an arc mode, which generally evolves into a constricted arc and develops hot spots on the electrode surfaces. The airflow and the magnetic field (from a ring-shaped permanent magnet) are introduced to prevent the discharge from forming arc constriction and hot spots. The airflow pushes the arc between the electrodes into an elongated loop (i.e., a gliding arc discharge), which extends out of the gap between electrodes more than $30 \mathrm{~mm}$. Magnetic field rotates the discharge loops to prevent the formation of hot spots on the electrodes. It stabilizes the discharge, slows down the build-up of the arc temperature, and reduces arc erosion on the electrodes.

Thus, the discharges are maintained in the elongated arc loop which rotates into a diffused cone over time. The increase of the discharge path length and the reduction of the transit time loss of the discharge work to significantly reduce the arc temperature, and the sparkling and heating of the electrodes, and to increase the plasma volume and the generation region of the reactive species, which is particularly important to those with short lifetimes, such as atomic oxygen.

\subsection{Emission Spectroscopy}

In the air discharge, where oxygen has lower ionization energy than that of the nitrogen, electrons $\left(\mathrm{e}^{-}\right)$and molecular oxygen ions $\left(\mathrm{O}_{2}^{+}\right)$are produced. As the air plasma flows out of the discharge zone, atomic oxygen (OI) is generated in the plasma effluent via a dissociative recombination process $\mathrm{O}_{2}^{+}+\mathrm{e}^{-} \rightarrow \mathrm{O}+\mathrm{O}$ as well as via other processes.

The presence of atomic oxygen in the plasma spray was examined via its emission spectroscopy [35] [36]. At atmospheric pressure, $777.4 \mathrm{~nm}$ and 844.5 $\mathrm{nm}$ lines are commonly found in non-equilibrium air discharges; $777.4 \mathrm{~nm}$ lines are usually more intense than $844.5 \mathrm{~nm}$ lines. O'Connor et al. [37] developed a method using the intensity ratio $\mathrm{I}_{844} / \mathrm{I}_{777}$ to determine the discharge gas temperature. In a scan of the emission spectroscopy as shown in Figure 1(b), the 777.4 $\mathrm{nm}$ lines have a dominant spectral peak, and the $844.5 \mathrm{~nm}$ lines are missing; it indicates that the discharge gas has low temperature. The $777.4 \mathrm{~nm}$ line is attributed to the $5 \mathrm{P}$ state of the atomic oxygen (OI). Presented in Figure 1(c) is the spatial distribution of the $777.4 \mathrm{~nm}$ radiation intensity ranging from $<10^{4}$ to $>10^{9}$ (in Rayleighs). The distribution extends out axially to about $25 \mathrm{~mm}$ from the nozzle of the module, where the intensity of the $777.4 \mathrm{~nm}$ line is about $10^{5} \mathrm{R}$. Thus, the apparent photon emission from a slice of the plasma plume at $25 \mathrm{~mm}$ away from the cap is about $10^{15} \mathrm{~m}^{-2} \cdot \mathrm{sec}^{-1}$, where the calculation assuming low optical thickness represents a minimum bound on the average flux.

\subsection{Surface Temperature during the Exposure to the Plasma Effluent}

This plasma spray is non-equilibrium due to the following factors associated with the design and the operation, 1) the discharge is run in a periodic mode with a duty cycle less than $10 \%, 2$ ) the airflow pushes the discharge along an 
elongated path, 3) the elongated arc loop is rotating by the magnetic field and vibrating by the air pressure in the nozzle, 4) the triggered discharge is maintained at low voltage. Because the discharge extends to 30 to $35 \mathrm{~mm}$ away from the discharge gap, some of the energetic electrons and OI are generated locally in the plasma plume, rather than being conveyed from the gap region of the electrodes. Thus, the thermal temperature $(<320 \mathrm{~K})$ of the plasma effluent is much lower than the excitation temperature of electrons channeled in the arc loop, which is estimated via emission spectroscopy to be higher than $7700 \mathrm{~K}$.

The temperature of an exposed surface is expected to increase with the treatment time. Agar is used to simulate the skin; it is then exposed to the plasma effluent, at an exposure distance of $30 \mathrm{~mm}$, continuously for up to 150 seconds. The surface temperature of the agar sample is then measured. As shown in Figure 2, it increases nearly linearly with the treatment time in the first 45 seconds, up from room temperature of $\sim 66^{\circ} \mathrm{F}\left(19^{\circ} \mathrm{C}\right)$ ) to about $100^{\circ} \mathrm{F}\left(38^{\circ} \mathrm{C}\right)$. After $45 \mathrm{~s}$, the temperature elevation rate drops quickly; the surface temperature of the agar sample increases to about $110^{\circ} \mathrm{F}\left(44^{\circ} \mathrm{C}\right)$ after $150 \mathrm{~s}$ exposure to the plasma effluent.

\section{Assay via Animal Model to Demonstrate CAAP Hemostasis Capacity}

In the following, two trials are presented. Two adult swines with blood pressure compatible to that of an adult human being, were used as animal models [38], and the similar trials were repeated on each swine. Each swine was first injected with calmative-stresnil and fastened on a table. It was then anesthetized with Isoflurance-Fluothane, which kept it in a narcotized state, and was monitored throughout the entire trial period.

\subsection{A Large, Curved Cut in Hind Quarters Area}

This large opening cut, shown in Figure 3(a), caused two broken arteries. Tourniquet was not applicable; instead, that using thumb to press above wound to slow down the active hemorrhage. Moreover, the cut arteries were hidden deeply inside the cut wound, where had to be opened and clearing away the blood with a medical cloth, to locate the arteries for treatment. In Figure 3(a), the handheld APS was moved back and forth around two cut arteries, holding a distance from each cut at about $30 \mathrm{~mm}$.

After about one-minute treatment, the wound was examined, but hemorrhage was not yet completely controlled. APS was then applied for another $45 \mathrm{~s}$. The second assessment of the wound, as indicated in Figure 3(b), confirmed that all hemorrhage was stopped completely. The total treatment time for this cut wound was about $105 \mathrm{~s}$.

\subsection{Amputation of a Front Leg}

The wound to be treated was a complete amputation of a front leg at the middle joint. This is a life-threatening hemorrhage situation; it involves three broken major arteries. 


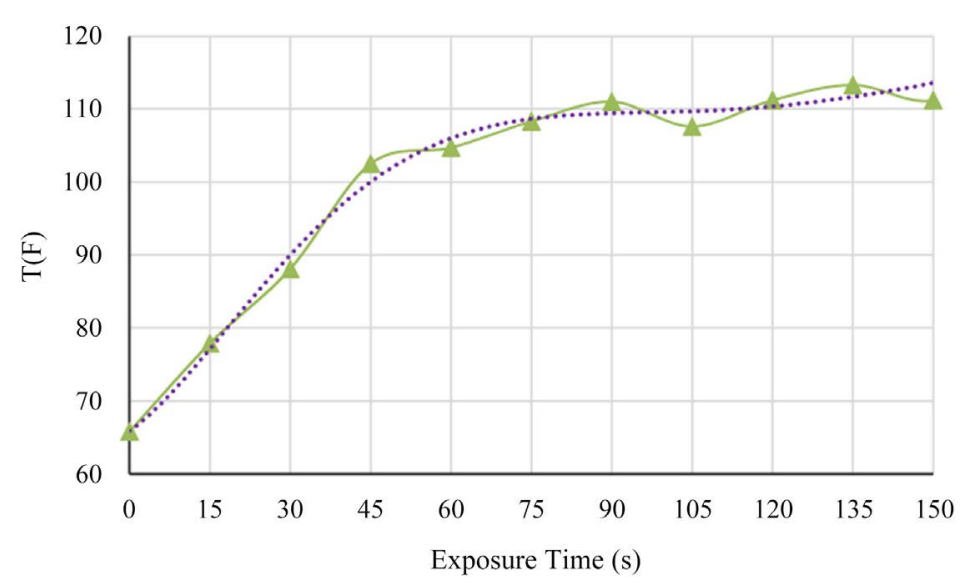

Figure 2. Dependency of the surface temperature of an agar sample on the treatment time to the plasma effluent at an exposure distance of $30 \mathrm{~mm}$.

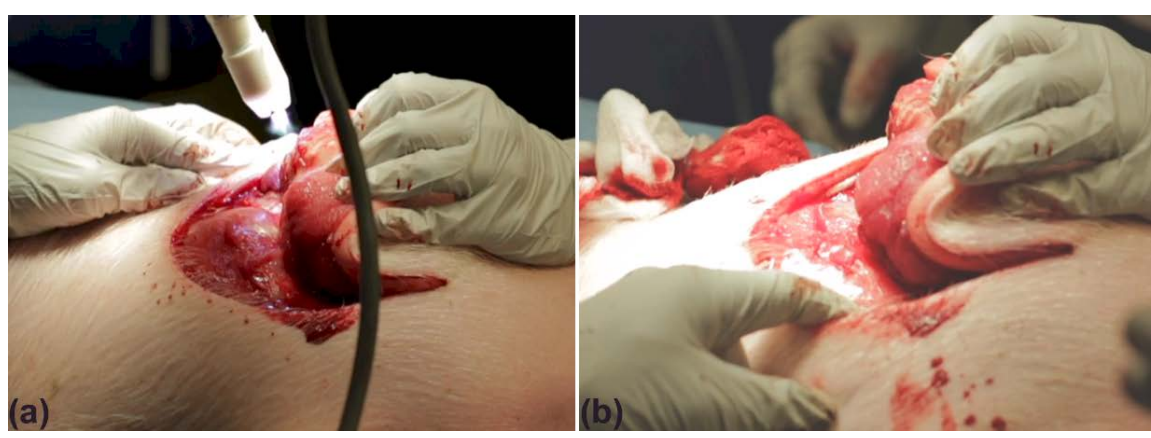

Figure 3. APS treatment on a large, curved cut wound in the hind quarters area; (a) treating the cut arteries back and forth continuously, holding at a distance from each cut around $30 \mathrm{~mm}$, and (b) assessing the wound to confirm no more bleeding after about 105 $s$ total treatment time.

As shown in Figure 4(a), tourniquet was applied in advance before performing the operation to slow down spurts of blood from the cut arteries; after the operation, APS was applied immediately to treat cut arteries as shown in Figure 4(b). In this location, the arteries are protected between bones, which makes difficult to constrict the cut arteries. Moving APS back and forth to cover three cut arteries and keeping around $30 \mathrm{~mm}$ from each cut in the treatment, it took about 5 minutes of continuous treatment to stop all hemorrhage, as shown in Figure 4(c). After the treatment, the tourniquet was loosened slowly to make sure that the wound did not re-bleed; the tourniquet was then removed, as shown in Figure 4(d). This is a demonstration of successful downgrade of a tourniquet-necessary wound. It meets the call for "platinum ten", i.e., achieving bleeding control within 10 minutes, to ensuring the survival of wounded personnel and mitigation of side effects. The wound can be simply wrapped with bandage for protection and preventing infection, before having a formal treatment in the hospital, as a protocol.

The capacity of APS in swift hemostasis and in downgrading hemostatic clamp/tourniquet necessary wounds has been demonstrated by the presented two trials. 


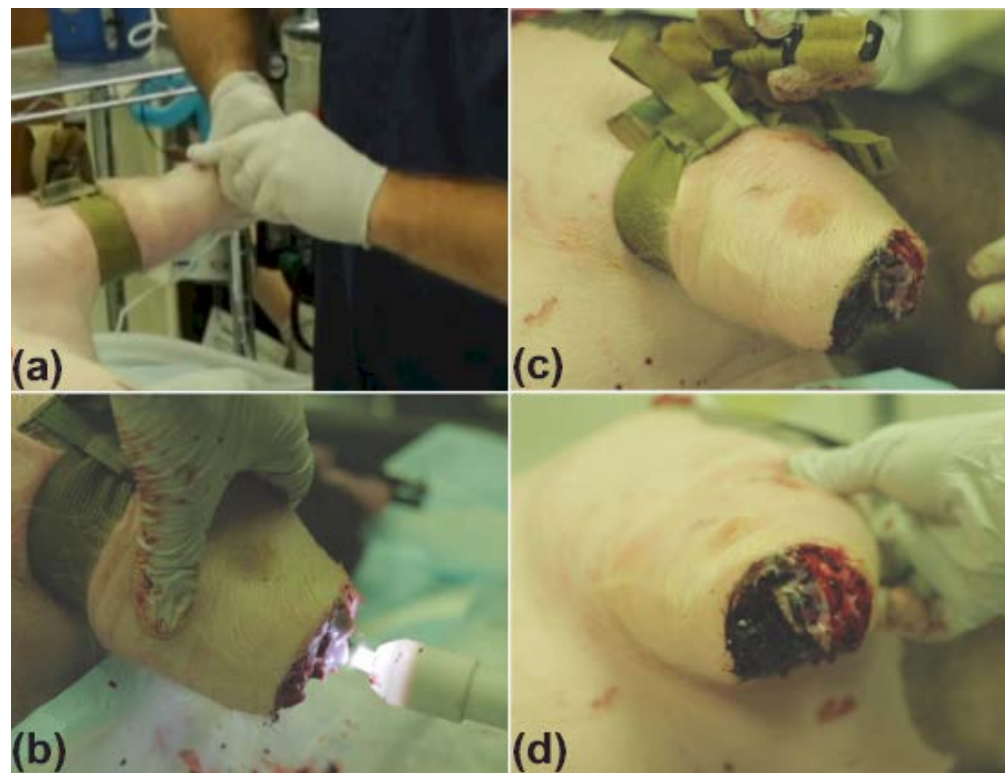

Figure 4. APS treatment on an amputated leg; (a) tourniquet is applied to slowdown hemorrhage from an amputated leg, (b) APS is applied to the cut arteries back and forth continuously, at a holding distance from each cut around $30 \mathrm{~mm}$, for more than 5 minutes treatment, (c) after all hemorrhage is controlled, tourniquet can be removed, and (d) wound is wrapped by bandage before giving a formal treatment in the hospital.

In the deep cut case of trial 1, there was no apparent red clots were observed after the bleeding was controlled. It suggests that the hemostasis is mainly through vasoconstriction accelerated by the ROS. When atomic oxygen interacts with $\mathrm{H}_{2} \mathrm{O}$, reactive oxygen species are produced to induce a procoagulant state in endothelial cells by altering tissue factor (TF) structure. ROS suppress NO's vasodilator activity to accelerate vasoconstriction and degrades plasmin in anticoagulation to enhance platelet aggregation, which then contract tissue to seal injured blood vessels.

Usually, hemostasis [39] comprises three major events that occur in a set order following the loss of vascular integrity:

1) vascular constriction and blood viscosity elevation to slow down bleeding,

2) platelets are activated by thrombin and change their shape. The protein fibrinogen aggregates platelets and stimulates platelet clumping by binding to collagen at the site of injury, forming a temporary, loose platelet plug, and

3) a fibrin mesh (also called the clot) forms and entraps the plug.

However, the blood pressure of the artery is too high to seal its cut by a blood clot from the outside; thus, the CAAP treatment also promotes:

4) vascular constriction and blood clotting right inside the cut (i.e., internal clot formation) to withstand the blood pressure of an artery.

\section{In-Vitro Tests to Explore Plasma Coagulation Mechanism}

Air plasma coagulation mechanism was explored by the tests, which were performed on the blood droplet samples and on the smeared blood samples with various CAAP treatments. Each test was repeated once. Blood samples used in 
tests were mixed with $3.2 \%$ sodium citrate solution at 9:1 ratio (in volume). The sodium citrate solution chelates calcium ions to prolong the natural clotting time [40] [41] to more than 25 minutes.

Blood is a fluid tissue that includes $60 \%$ of a liquid portion known as blood plasma, and $40 \%$ of formed elements or blood cells [42]. The formed elements and the blood plasma involve in different ways in contributing blood coagulation during hemorrhage; to single out the direct relevance of CAAP in promoting coagulation cascade, a precipitation process is then applied to separate whole blood into blood plasma and formed elements, which are also used to prepare droplet samples in the tests.

\subsection{Blood-Droplet Tests}

Shown in row 1 of Figure 5 are the untreated droplets set on glass slides, used as the controls of (a) whole blood, (b) blood plasma, (c) formed elements, and (d) whole blood, droplet-samples. The treated sampled are presented in row 2 to row 4. The treatment time is $16 \mathrm{~s}$; the exposure distance decreases from $40 \mathrm{~mm}$ (row 2) to $30 \mathrm{~mm}$ (row 3), and then to $25 \mathrm{~mm}$ (row 4). The first whole blood sample in (1a) was treated by the hot airflow of a hair dryer, which is hotter than that of the plasma flow. Photos of the whole blood sample taken after $16 \mathrm{~s}$ of this hot air treatment at three exposure distances of $40 \mathrm{~mm}, 30 \mathrm{~mm}$, and $25 \mathrm{~mm}$, are presented in $(2 \mathrm{a})$ to $(4 \mathrm{a})$.

\begin{tabular}{|c|c|c|c|c|}
\hline $16 \mathrm{sec}$ & $\begin{array}{l}\text { Whole blood } \\
\text { (fan only) } \\
\text { (a) }\end{array}$ & $\begin{array}{l}\text { platelet poor } \\
\text { (b) }\end{array}$ & $\begin{array}{l}\text { platelet rich } \\
\text { (c) }\end{array}$ & $\begin{array}{l}\text { Whole blood } \\
\text { (d) }\end{array}$ \\
\hline Control & & & & \\
\hline $40 \mathrm{~mm}$ & & & & \\
\hline (2) & & e & & \\
\hline $30 \mathrm{~mm}$ & & & & \\
\hline (3) & & & & \\
\hline $25 \mathrm{~mm}$ & & & & \\
\hline (4) & & & & \\
\hline
\end{tabular}

Figure 5. Untreated controls (row 1) of whole blood (1a), blood plasma (1b), formed elements (1c), and whole blood (1d, the same as 1a), droplet-samples; and the corresponding hot air treated samples $(2 \mathrm{a}-4 \mathrm{a})$ and plasma treated samples $(2 \mathrm{~b}-4 \mathrm{~b}, 2 \mathrm{c}-4 \mathrm{c}$, and $2 \mathrm{~d}-4 \mathrm{~d})$ with $16 \mathrm{~s}$ treatment time and with three exposure distances of $40 \mathrm{~mm}, 30 \mathrm{~mm}$, and $25 \mathrm{~mm}$. 
The first whole blood sample in (1a) was treated by the hot airflow of a hair dryer, which is hotter than that of the plasma flow. Photos of the whole blood sample taken after $16 \mathrm{~s}$ of this hot air treatment at three exposure distances of 40 $\mathrm{mm}, 30 \mathrm{~mm}$, and $25 \mathrm{~mm}$, are presented in (2a) to (4a) of Figure 5. It shows that the heat of the airflow can quickly strengthen the shell of the droplet to withhold the blow of the airflow; it raises the internal pressure of the blood droplet, which pushes blood to the center to be expanded upward. However, the heat of the airflow did not instigate any blood clotting in all cases of different exposure distance.

The other three controls in row 1 of Figures 5(b)-5(d) were treated by CAAP. The test results are presented in rows 2 to 4 of Figures 5(b)-5(d) for treated (b) blood plasma, (c) formed elements, and (d) whole blood, samples; sample temperatures were raised by the plasma flow (see Figure 2), but much less than that raised by the hot airflow of a hair dryer. A dark brown shell, manifesting blood clotting, can be seen in the formed elements samples (2c)-(4c) and whole blood samples (2d)-(4d), but it is not observed in the treated blood plasma samples (2b)-(4b).

The treated formed elements sample (2c)-(4c) is darker, dryer, and harder than the corresponding treated whole blood samples (2d)-(4d). The results presented in columns $\mathrm{c}$ and $\mathrm{d}$ also show that the degree of clotting of treated formed elements samples and whole blood samples increases with the decrease of the exposure distance.

Heat can induce protein coagulation; on the other hand, applying hotter airflow (than the heat flow of the plasma effluent), there is no indication of clotting in the treated whole blood samples (2a)-(4a) as well as in the treated blood plasma samples (2b)-(4b). It evidences that the heat delivered by the CAAP cannot cause blood clotting in the CAAP treatment. Moreover, the blood plasma samples, in which the form elements are removed, also cannot be clotted by the CAAP.

Thus, the clotting of the treated samples in (2c)-(4c) and (2d)-(4d) is ascribed to the activation of the formed elements by the reactive species; the plasma effluent delivers more reactive species in shorter exposure distance treatment. The results clearly demonstrate the coagulation efficacy of the CAAP applied in the tests, which shortens the clotting time from 25 minutes extent to 16 second margin.

CAAP treatments with different exposure distance and time on each blood droplet sample, which was set on a glass slide, were performed to correlate the OI flux with the degree of clotting. The total amount of OI applied to the blood droplet sample in treatment will be proportional to the total treatment time and inversely proportional to the exposure distance. Presented in Figure 6(a) is an untreated control. Three samples treated at the same exposure distance of 25 $\mathrm{mm}$ for 8,12 , and $16 \mathrm{sec}$, respectively, are presented in Figures 6(b)-6(d) for comparison. A shell, formed on each blood sample surface, can be clearly seen. The photos indicate that the degree of blood clotting increases with the increase 

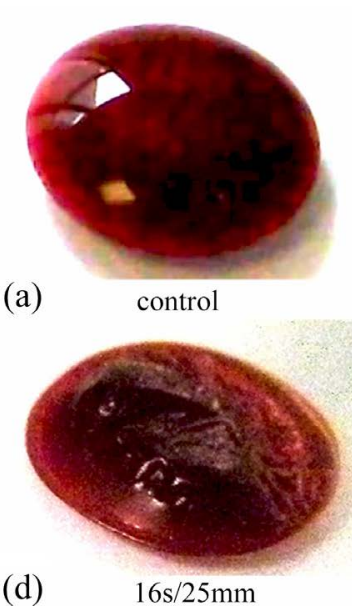

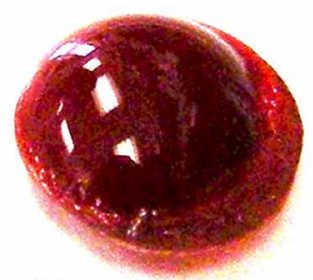

(b)

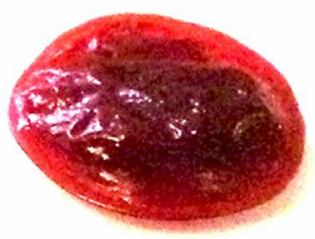

(e) $16 \mathrm{~s} / 30 \mathrm{~mm}$

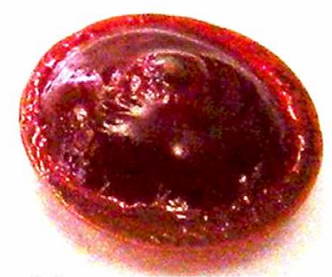

(c)

$12 \mathrm{~s} / 25 \mathrm{~mm}$

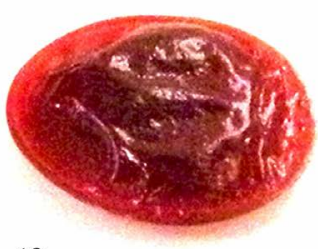

(f)

$16 \mathrm{~s} / 40 \mathrm{~mm}$

Figure 6. Blood samples: (a) untreated control, (b) to (d) treated by the CAAP at a fixed exposure distance of $25 \mathrm{~mm}$ with increasing treatment times of (b) $8 \mathrm{sec}$, (c) $12 \mathrm{sec}$, and (d) $16 \mathrm{sec}$, and (d) to (f) treated by the CAAP with a fixed treatment time of $16 \mathrm{sec}$ at three exposure distances of (d) $25 \mathrm{~mm}$, (e) $30 \mathrm{~mm}$, and (f) $40 \mathrm{~mm}$.

of the treatment time from 8 to $16 \mathrm{sec}$. Three samples treated at three different exposure distances of 25,30 , and $40 \mathrm{~mm}$ for the same treatment time of $16 \mathrm{sec}$ are presented in Figures 6(d)-6(f). As shown, the degree of blood clotting decreases as the exposure distance increases from 25 to $40 \mathrm{~mm}$.

The critical role of atomic oxygen/ROS in enhancing coagulation can be further evidenced by comparing the coagulation time of cold atmospheric plasma (CAP) treatment by different plasma devices employing different working gases. The kINPen Plasma MED [24] is an atmospheric pressure plasma jet (APPJ) using Argon gas as the working gas. It generates ROS only after encountering the ambient air outside the generator. It takes 10 minutes to achieve a complete clot. The air plasma of a DBD device can only initiate noticeable partial coagulation on normal blood droplet samples (without mixing with coagulation agents) in 15 seconds [25], down from the natural clotting time of about 30 seconds. On the other hand, an anticoagulation blood droplet sample reaches a complete clot by the CAAP treatment (by delivering abundance of OI) in 16 seconds.

\subsection{Tests on Smeared Blood Samples}

Untreated (control) and CAAP-treated smeared whole blood samples were prepared for cell staining and microscopy analysis, which identified cell types and performed cell counts.

Presented in Figure 7 are the results of RBC and platelet cell counts, from smeared whole blood control and from treated samples with four different treatment times of 2, 3, 4, and $10 \mathrm{~s}$ at $25 \mathrm{~mm}$ exposure distance; and from a smeared whole blood sample treated at $40 \mathrm{~mm}$ exposure distance with $10 \mathrm{~s}$ treatment time.

It is shown that the smeared whole blood samples treated at a fixed exposure distance of $25 \mathrm{~mm}$, the concentration of RBC/platelets increases/decreases monotonically as the treatment time increases. Cell counts of RBC and platelets 


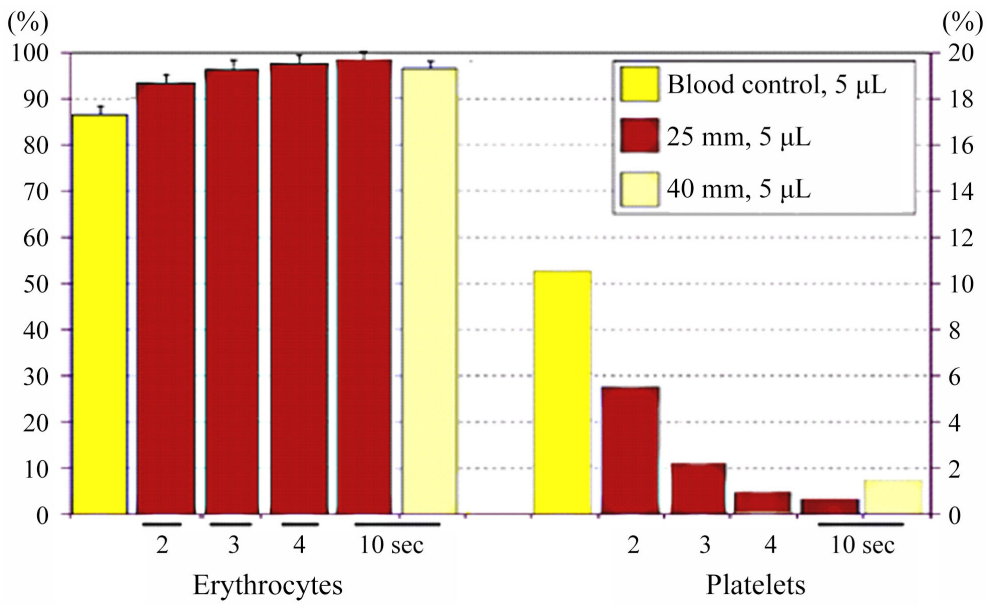

Figure 7. Variation of the RBC and platelet concentrations of smeared whole blood samples as the treatment time increased from $2 \mathrm{~s}$ to $10 \mathrm{~s}$ and the exposure distance increased from $25 \mathrm{~mm}$ to $40 \mathrm{~mm}$.

with $10 \mathrm{~s}$ treatment time at $25 \mathrm{~mm}$ and $40 \mathrm{~mm}$ exposure distances were compared.

The results show that the treatment at longer exposure distance causes less changes (i.e., less increase/decrease) on the concentrations of RBC and platelets. These test results evidence that CAAP activates RBC and platelets; an increasing of delivered OI by the CAAP effluent causes the decreasing of the platelet count (i.e., increasing of activated platelet count) and the increasing of RBC count in the treated samples.

The results of the tests on blood droplet samples presented in Figure 6 and the microscopy analyses of smeared whole blood samples presented in Figure 7 evidence that the rising of clotting by increasing plasma treatment correlates with the increasing/decreasing of $\mathrm{RBC} /$ platelet concentration counts. These dependencies are also correlated to the increasing of applied OI in the treatment (by decreasing the exposure distance). It infers that OI carried by the plasma effluent could rapidly trigger coagulation cascade.

\subsection{A Plausible Mechanism}

Imitated plots presented in Figure 8 incorporate the correlations deduced from the test results, which include the dependencies of the degree of clotting, the RBC count, and the platelet count, on the amount of atomic oxygen supplied in the CAAP treatment. The sequence of the plots illustrates a plasma clotting mechanism, which is described in the following.

Atomic oxygen is very reactive and can oxide most elements. It is essential for respiration of animals and can be used in medical applications. It kills disease microorganisms and improve cellular function. The test results further reveal its significant function of promoting hemostasis. When atomic oxygen (OI) interacts with $\mathrm{H}_{2} \mathrm{O}$ in the blood plasma, $\mathrm{H}_{2} \mathrm{O}_{2}$ and $\mathrm{OH}^{*}$ are produced. These reactive oxygen species are expected to function similarly to those oxidants produced or 


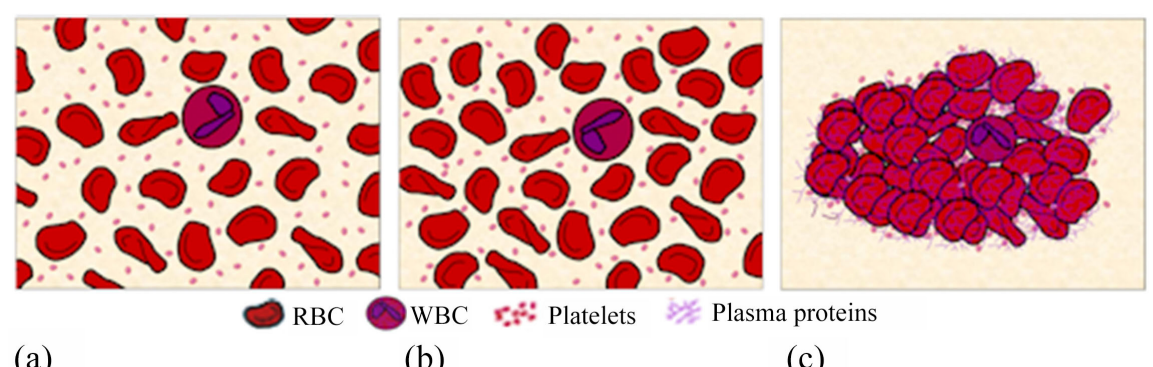

(a)

(b)

(c)

Figure 8. Imitated plots illustrate the clotting process in the CAAP treatment.

released in the vascular lumen. Several key steps in coagulation cascade are then triggered. The stimulated RBC-platelets and WBC interactions (Figure 8(a)) influence the concentration of cells suspended in blood (Figure $8(\mathrm{~b})$ ). The observations of the microscope presented in Figure 7 show the increase of RBC concentration in line with the increase of the treatment time. Enhanced adenine nucleotides released by aggregated $\mathrm{RBC}$ trigger platelet adherence/agglomeration [40] [43]-[48], where fibrinogen acts as a bridge to link activated platelets together. Atomic oxygen also indirectly activates and aggregate platelets by inactivating anticoagulation agents, such as endothelial-derived relaxing factor (EDRF) which counteracts platelet aggregation to control clotting. Subsequently, globular complexes (Figure 8(a) and Figure 8(b)) are formed to trap RBC and activated platelets, leading to the decrease of inactivated platelet concentration; the viscosity of blood samples will also be affected by atomic oxygen and oxidants that presumably contribute to the denaturation of albumin [41] as well as other proteins found in the blood. Consequently, the blood fluid identity decreases, and coagulation rises. Additionally, oxidant-activated platelets release the contents of stored granules into the blood plasma to induce thrombin; it converts the soluble fibrinogen, a large and complex glycoprotein, into insoluble fibrin strands; fibrin gel glues activated platelets together as hemostatic plug, which is then covered by fibrin mesh to form clot (Figure 8(c)).

\section{Summary of CAAP Hemostasis Mechanism}

The in-vivo trial results presented in Section 3 have demonstrated that this CAAP spray rapidly stopped hemorrhage from life-threatening wounds. In Section 2, the emission spectroscopy indicates that CAAP effluent carries atomic oxygen. In Section 4, test results on blood droplet samples and on the smeared blood samples deduce that atomic oxygen is the dominant factor instigating coagulation cascade.

Hemostasis [39] comprises three major events that occur in a set order following the loss of vascular integrity:

1) Vascular constriction and blood viscosity elevation to slow down bleeding.

2) Platelets are activated by thrombin and change their shape. The protein fibrinogen aggregates platelets and stimulates platelet clumping by binding to collagen at the site of injury, forming a temporary, loose platelet plug. 
3) A fibrin mesh (also called the clot) forms and entraps the platelet plug.

In the treatment, the atomic oxygen (OI) delivered by the CAAP interacts with water $\left(\mathrm{H}_{2} \mathrm{O}\right)$ content in the blood plasma, oxidants are produced. Oxidants alter tissue factor pathway inhibitor (TFPI) structure that a pro-coagulant state in endothelial cells is induced. They neutralize nitric oxide (NO) to reverse vasodilator activity and regulate plasminogen-induced IL- $1 \beta$ and TNF- $\alpha$ production in microglia to degrade plasmin in anticoagulation. The viscosity of blood is affected by oxidants that presumably contribute to the denaturation of albumin and other proteins found in the blood. Consequently, the blood fluid identity decreases to slow down bleeding. These oxidants also target platelets, in the same way as those produced or released in the vascular lumen, to affect several key steps of platelet function, such as platelet activation, aggregation, and adhesion.

The activated platelets release the contents of stored granules into the blood plasma and change shape from spherical to stellate; and the fibrinogen cross-links with platelets' glycoprotein to aggregate adjacent platelets, which are then bound to the collagen exposed on endothelial cell surfaces. A glycoprotein called von Willebrand factor (vWF), which is found in blood plasma, further strengthens this adhesion by binding collagen to the platelets; this binding activates platelet integrins, which mediate tight binding of platelets to the extracellular matrix and thus adhere this platelet plug (white clot) to the site of injury; this "primary hemostasis" process is accelerated significantly by the OI flux delivered by the CAAP.

$\mathrm{H}_{2} \mathrm{O}_{2}$, produced by OI, stimulates phospholipase A2 enzyme to amplify platelet response to collagen and acts as second messenger by activating arachidonic acid metabolism and phospholipase C (PLC) pathway [15]. It rapidly advances platelet aggregation to set off an increase in the release of $\mathrm{C}_{\mathrm{a}}^{2+}$ ions, which, together with thrombokinase/thromboplastin (factor III) released by the damaged tissues, react with prothrombin to form thrombin. Thrombin converts soluble fibrinogen into insoluble fibrin to deposit into the platelet plug and activates platelets to mediate the formation of covalent bonds, which crosslink the fibrin polymers to form fibrin mesh (red clot) all around the platelet plug to hold it in place. The supplied OI flux also speeds up this "secondary hemostasis" process.

However, the blood pressure of the artery is too high to seal its cut by a blood clot from the outside; thus, in the CAAP treatment, vascular constriction and blood clotting right inside the cut (i.e., internal clot formation) are likely the mechanism of sealing cut arteries in large cut wounds, such as those shown in Figure 3 and Figure 4.

In sum, this CAAP is effectual to trigger various biochemical reactions to promote hemostasis. It speeds up vascular constriction, activates platelets, and instigates the crosslinks of various blood polymers to set off coagulation cascade, which boost hemostasis as demonstrated by the two trials presented in Section 3 .

\section{Discussion and Conclusion}

The time that has elapsed since the wound occurred is an important factor in 
determining whether the wound should be closed primarily. Fast bleeding control together with proper disinfection in the treatment will allow some wounds to be closed quickly without an increased risk of infection; that can reduce the patient's discomfort, speed wound healing, and decrease scarring.

Some injuries can cause a trauma patient to deteriorate rapidly, the time delay between injury and treatment must be kept to a minimum. It is well established that the patient's chances of survival are greatest if they receive definitive care within a short period of time after a severe injury [49].

The golden hour represents the span of time in which treatment of bleeding offers the greatest hope of survival [50]. Moreover, it is also called for a full control of hemorrhage within as few as 10 minutes (so-called platinum ten) to ensure the survival of wounded persons in accidents, as well as to minimizing the risk of complications in the recovery.

Thus, it is desirable that the critical traumatic patients in the emergency are treated with effective first aid [5], which is capable to stop main hemorrhage of the wound within the initial 10 minutes period and preventing infection. It is the preference that the first aid tool is portable on the battlefield and at accident sites.

The effectiveness of this CAAP in rapid control of active hemorrhage has been demonstrated (Figure 3 and Figure 4). The biocidal effects of the CAAP [19] [31] [32] [33] also provide disinfection simultaneously in the treatment. Further tests on wounds of artery cuts at different locations will be useful to establish the treatment procedure in the first aid in any situation. A CAAP device, which can be made portable by using battery as the power source [51], should meet the requirements of advanced first aid [52].

Finally, atomic oxygen together with generated ROS (such as ${ }^{\star} \mathrm{OH}$ and $\mathrm{H}_{2} \mathrm{O}_{2}$ ), via interacting with $\mathrm{H}_{2} \mathrm{O}$, work for disinfection. Through oxidation-reduction reactions, oxygen radicals kill bacteria by denaturation of proteins in cell walls outside the cores of bacteria, to shorten the period of the inflammatory phase in wound healing. Moreover, those radicals affect eukaryotic cells, especially on keratinocytes in terms of viability, proliferation, adhesion molecules and angiogenesis. Thus, this CAAP also accelerates wound healing, by its antiseptic effects, by stimulation of proliferation and migration of wound relating skin cells, by activation or inhibition of integrin receptors on the cell surface or by its pro-angiogenic effect.

\section{Acknowledgements}

The author is grateful to Alessandro Betti and Edmund Chow for helping me to develop the air plasma generator used in the experiments, and to Olga Tarasenko, Cheng-Yen Chen, Chuan-Shun Lin, Todd Pedersen, Zach Steinbock, and Sean Matson for different helps and advice. This work was supported in part by the Adventix Technologies Inc. and the Aceso Plasma, LLC.

\section{Conflicts of Interest}

The author declares no conflicts of interest regarding the publication of this paper. 


\section{References}

[1] Spinella, P.C., Perkins, J.G., McLaughlin, D.F., Niles, S.E., Grathwohl, K.W., Beekley, A.C., Salinas, J., Mehta, S., Wade, C.E. and Holcomb, J.B. (2008) The Effect of Recombinant Activated Factor VII on Mortality in Combat-Related Casualties with Severe Trauma and Massive Transfusion. The Journal of Trauma: Injury, Infection, and Critical Care, 64, 286-294. https://doi.org/10.1097/TA.0b013e318162759f

[2] Perkins, J.G., Cap, A.P., Weiss, B.M., Reid, T.J. and Bolan, C.D. (2008) Massive Transfusion and Nonsurgical Hemostatic Agents. Critical Care Medicine, 36, S325-S339.

[3] Milovanov, A.P. and Kirsanov, Ia.N. (2008) The Pathogenesis of Uterine Hemorrhages in the So-Called Placental Polyps. Arkhiv Patologii, 70, 34-37.

[4] Kramer, A.H., Gurka, M.J., Nathan, B., Dumont, A.S., Kassell, N.F. and Bleck, T.P. (2008) Complications Associated with Anemia and Blood Transfusion in Patients with Aneurysmal Subarachnoid Hemorrhage. Critical Care Medicine, 36, 2070-2075.

[5] Jevon, P. and Cooper, L. (2008) First Aid. Part 5: First-Aid Treatment for Severe Bleeding. Nursing Times, 104, 26-27.

[6] Scharf, R.E. (2008) Acquired Platelet Function Disorders: Pathogenesis, Classification, Frequency, Diagnosis, Clinical Management. Hamostaseologie, 28, 299-311.

[7] Trouillas, P. and von Kummer, R. (2006) Classification and Athogenesis of Cerebral Hemorrhages after Thrombolysis in Ischemic Stroke. Stroke, 37, 556-561. https://doi.org/10.1161/01.STR.0000196942.84707.71

[8] Franchini, M. (2008) Surgical Prophylaxis in Von Willebrand's Disease: A Difficult Balance to Manage. Blood Transfusion, 6, s33-s38.

[9] Al-Sallami, H., Ferguson, R., Wilkins, G., Gray, A. and Medlicott, N.J. (2008) Bleeding Events in Patients Receiving Enoxaparin for the Management of Non-ST-Elevation Acute Coronary Syndrome (NSTEACS) at Dunedin Public Hospital, New Zealand. New Zealand Medical Journal, 121, 87-95.

[10] Roy, P., Bonello, L., de Labriolle, A., Lemesle, G., Pinto Slottow, T.L., Steinberg, D.H., et al. (2008) Impact of "Nuisance" Bleeding on Clopidogrel Compliance in Patients Undergoing Intracoronary Drug-Eluting Stent Implantation. American Journal of Cardiology, 102, 1614-1617. https://doi.org/10.1016/j.amjcard.2008.07.063

[11] Jamal, M.M., Samarasena, J.B. and Hashemzadeh, M. (2008) Decreasing in-Hospital Mortality for Oesophageal Variceal Hemorrhage in the USA. European Journal of Gastroenterology \& Hepatology, 20, 947-955. https://doi.org/10.1097/MEG.0b013e32830280c7

[12] Thanvi, B.R., Treadwell, S. and Robinson, T. (2008) Haemorrhagic Transformation in Acute Ischaemic Stroke Following Thrombolysis Therapy: Classification, Pathogenesis and Risk Factors. Postgraduate Medical Journal, 84, 361-367. https://doi.org/10.1136/pgmj.2007.067058

[13] Derex, L. and Nighoghossian, N. (2008) Intracerebral Haemorrhage after Thrombolysis for Acute Ischaemic Stroke: An Update. Journal of Neurology, Neurosurgery \& Psychiatry, 79, 1093-1099. https://doi.org/10.1136/jnnp.2007.133371

[14] Ambrosio, G., Tritto, I. and Golino, P. (1997) Reactive Oxygen Metabolites and Arterial Thrombosis. Cardiovascular Research, 34, 445-452. https://doi.org/10.1016/S0008-6363(97)00101-6

[15] Del Principe, D., Menichelli, A., De Matteis, W., Di Giulio, S., Giordani, M., Savini, I. and Finazzi-Agro, A. (1991) Hydrogen Peroxide Is an Intermediate in the Platelet Activation Cascade Triggered by Collagen, But not by Thrombin. Thrombosis Re- 
search, 62, 365-375. https://doi.org/10.1016/0049-3848(91)90010-T

[16] Del Principe, D., Menichelli, A., De Matteis, W., Di Corpo, M.L, Di Giulio, S. and Finazzi-Agro, A. (1985) Hydrogen Peroxide has a Role in the Aggregation of $\mathrm{Hu}-$ man Platelets. FEBS Letters, 185, 142-146. https://doi.org/10.1016/0014-5793(85)80758-4

[17] Ambrosio, G., Oriente, A., Napoli, C., Palumbo, G., Chiariello, P., Marone, G., Condorelli, M., Chiariello, M. and Triggiani, M. (1994) Oxygen Radicals Inhibit Human Plasma Acetylhydrolase, the Enzyme that Catabolizes Platelet-Activating Factor. Journal of Clinical Investigation, 93, 2408-2416.

https://doi.org/10.1172/JCI117248

[18] Golino, P., Ragni, M., Cirillo, P., Avvedimento, V.E., Feliciello, A., Esposito, N., et al. (1996) Effects of Tissue Factor Induced by Oxygen Free Radicals on Coronary Flow during Reperfusion. Nature Medicine, 2, 35-40. https://doi.org/10.1038/nm0196-35

[19] Kuo, S. (2019) Cold Atmospheric Plasmas: Their Use in Biology and Medicine. World Scientific, Singapore. https://doi.org/10.1142/11264

[20] Stoffels, E., Flikweert, A.J., Stoffels, W.W. and Kroesen, G.M.W. (2002) Plasma Needle: A Non-Destructive Atmospheric Plasma Source for Fine Surface Treatment of (Bio) Materials. Plasma Sources Science and Technology, 11, 383. https://doi.org/10.1088/0963-0252/11/4/304

[21] Kogelschatz, U. (2003) Dielectric-Barrier Discharges: Their History, Discharge Physics and Industrial Applications. Plasma Chemistry and Plasma Processing, 23, 1-46. https://doi.org/10.1023/A:1022470901385

[22] Laroussi, M. (2015) Low Temperature Plasma Jet for Biomedical Applications: A Review. IEEE Transactions on Plasma Science, 43, 703-711. https://doi.org/10.1109/TPS.2015.2403307

[23] Laroussi, M. (2002) Non-Thermal Decontamination of Biological Media by Atmospheric Pressure Plasmas: Review, Analysis, and Prospects. IEEE Transactions on Plasma Science, 30, 1409-1415. https://doi.org/10.1109/TPS.2002.804220

[24] Bekeschus, S., Schmidt, A., Weltmann, K. D. and Woedtke, T. (2016) The Plasma Jet kINPen-A Powerful Tool for Wound Healing. Clinical Plasma Medicine, 4, 19-28. https://doi.org/10.1016/j.cpme.2016.01.001

[25] Fridman, G., Peddinghaus, M., Balasubramanian, M., Ayan, H., Fridman, A., Gutsol, A. and Brooks, A. (2006) Blood Coagulation and Living Tissue Sterilization by Floating-Electrode Dielectric Barrier Discharge in Air. Plasma Chemistry and Plasma Processing, 26, 425-442. https://doi.org/10.1007/s11090-006-9024-4

[26] Fridman, G., Brooks, A.D., Balasubramanian, M., Fridman, A., Gutsol, A., Vasilets, V.N., Ayan, H. and Friedman, G. (2007) Comparison of Direct and Indirect Effects of Non-Thermal Atmospheric-Pressure Plasma on Bacteria. Plasma Processes and Polymers, 4, 370-375. https://doi.org/10.1002/ppap.200600217

[27] Lai, W., Lai, H., Kuo, S.P., Tarasenko, O. and Levon, K. (2005) Decontamination of Biological Warfare Agents by a Microwave Plasma Torch. Physics of Plasmas, 12, Article ID: 023501. https://doi.org/10.1063/1.1843131

[28] Chen, C.Y., Fan, H.W., Kuo, S.P., Chang, J., Pedersen, T., Mills, T. and Huang, C.C. (2009) Blood Clotting by Low Temperature Air Plasma. IEEE Transaction on Plasma Science, 37, 993-999. https://doi.org/10.1109/TPS.2009.2016344

[29] Kuo, S.P., Tarasenko, O., Chang, J., Popovic, S., Chen, C., Fan, H., Scott, A., Lahiani, M., Alusta, P., Drake, J. and Nikolic, M. (2009) Contribution of a Portable Air Plasma Torch to Rapid Blood Coagulation as a Method of Preventing Bleeding. 
New Journal of Physics, 11, Article ID: 115016.

https://doi.org/10.1088/1367-2630/11/11/115016

[30] Kuo, S.P., Chen, C.Y., Lin, C.S. and Chiang, S.H. (2010) Wound Bleeding Control by Low Temperature Air Plasma. IEEE Transaction on Plasma Science, 38, 1908-1914. https://doi.org/10.1109/TPS.2010.2047028

[31] Kuo, S.P., Chen, C.Y., Lin, C.S. and Chiang, S.H. (2012) Applications of Air Plasma for Wound Bleeding Control and Healing. IEEE Transaction on Plasma Science, 40, 1117-1123. https://doi.org/10.1109/TPS.2012.2184142

[32] Duarte, S., Kuo, S.P., Murata, R.M., Chen, C.Y., Saxena, D., Huang, K.J. and Popovic, S. (2011) Air Plasma Effect on Dental Disinfection. Physics of Plasmas, 18, Article ID: 073503, 7 p. https://doi.org/10.1063/1.3606486

[33] Kuo, S.P. (2012) Air Plasma for Medical Applications. Journal of Biomedical Science and Engineering, 5, 481-495. http://www.scirp.org/journal/jbise/ https://doi.org/10.4236/jbise.2012.59061

[34] Kuo, S.P. (2010) Portable Plasma Sterilizer. US Patent No. 7777151 B2.

[35] Kuo, S.P., Pedersen, T. and Mills, T. (2008) Lateral Distribution of Atomic Oxygen Flux Produced by an Array of Three Fan-Shaped Plasma Torches. IEEE Transaction on Plasma Science, 36, 1056-1057. https://doi.org/10.1109/TPS.2004.924556

[36] Kuo, S.P., Pedersen, T. and Mills, T. (2011) Two-Dimensional Distribution of Atomic Oxygen Multiplet Radiation Produced by an Air Plasma Torch. IEEE Transaction on Plasma Science, 39, 2282-2283. https://doi.org/10.1109/TPS.2011.2155089

[37] O'Connor, N., Humphreys, H.H. and Daniels, S. (2013) Oxygen Line Ratio Method for the Determination of Plasma Parameters in Atmospheric Pressure Discharges using Air as the Working Gas. 31 th International Conference on Phenomena in Ionized Gases (ICPIG), Granada, 14-19 July 2013, 2-5.

[38] Kuo, S. (2018) On Hemostasis of Cold Atmospheric Air Plasma. Open Journal of Emergency Medicine, 6, 99-111. https://doi.org/10.4236/ojem.2018.64012

[39] Boon, G.D. (1993) An Overview of Hemostasis. Toxicologic Pathology, 21, 170-179. https://doi.org/10.1177\%2F019262339302100209

[40] Kolev, K. and Longstaff C. (2016) Bleeding Related to Disturbed Fibrinolysis. British Journal of Haematology, 175, 12-23. https://doi.org/10.1111/bjh.14255

[41] Meireles, M., Aimar, P. and Sanchez, V. (2004) Albumin Denaturation during Ultrafiltration: Effects of Operating Conditions and Consequences on Membrane Fouling. Biotechnology and Bioengineering, 38, 528-534.

https://doi.org/10.1002/bit.260380511

[42] Born, G.V.R., Bergquist, D. and Arfors, K.E. (1976) Evidence for Inhibition of Platelet Activation in Blood by a Drug Effect on Erythrocytes. Nature, 259, 233-235. https://doi.org/10.1038/259233a0

[43] Machovich, R. and Owen, W.G. (1990) The Elastase-Mediated Pathway of Fibrinolysis. Blood Coagulation \& Fibrinolysis, 1, 79-90.

https://doi.org/10.1097/00001721-199003000-00011

[44] Bergquist, D. and Arfors, K.E. (1980) Haemostatic Platelet Plug Formation in the Isolated Rabbit Mesenteric Preparation-An Analysis of Red Blood Cell Participation. Thrombosis and Haemostasis, 44, 6-8. https://doi.org/10.1055/s-0038-1650069

[45] Schmid-Schdnbein, H. and Teitel, P. (Eds.) (1979) Basic Aspects of Blood Trauma. Springer, Dordrecht, 322-340. https://doi.org/10.1007/978-94-009-9337-2

[46] Tiefenbach, H.J., Durchschlag, H., Schneider, G. and Jaenicke, R. (2004) Thermodynamic Analysis of Serum Albumin Denaturation by Sodium Dodecyl Sulfate. In: 
Anghel, D.F., Ed., Aqueous Polymer-Cosolute Systems, Vol. 124, Springer, Berlin, Heidelberg. 130-140. https://doi.org/10.1007/3-540-36114-6_16

[47] Schmid-Schdnbein, H., Born, G.V.R., Richardson, P.D., Cusack, N., Rieger, H., Forst, R., Rohling-Winkel, J., Blasberg, P. and Wehmeyer, A. (1981) Rheology of Thrombotic Processes in Flow: The Interaction of Erythrocytes and Thrombocytes Subjected to High Flow Forces. Biorheology, 18, 415-444.

https://doi.org/10.3233/BIR-1981-183-609

[48] Massberg, S., Grahl, L., von Bruehl, M., Manukyan, D., Pfeiler, S., Goosmann, C., et al. (2010) Reciprocal Coupling of Coagulation and Innate Immunity via Neutrophil Serine Proteases. Nature Medicine, 16, 887-896. https://doi.org/10.1038/nm.2184

[49] American College of Surgeons (2008) ATLS, Advanced Trauma Life Support Program for Doctors. American College of Surgeons, Chicago.

[50] Lerner, E.B. and Moscati, R.M. (2001) The Golden Hour: Scientific Fact or Medical "Urban Legend"? Academic Emergency Medicine, 8, 758-760. https://doi.org/10.1111/j.1553-2712.2001.tb00201.x

[51] Kuo, S.P. (2015) Battery Powered Handheld Air Plasma Spray. US Patent No. 8927896 B2.

[52] Kuo, S. (2016) Air Plasma Spray for First Aid. Open Journal of Emergency Medicine, 4, 69-82. https://doi.org/10.4236/ojem.2016.43010 